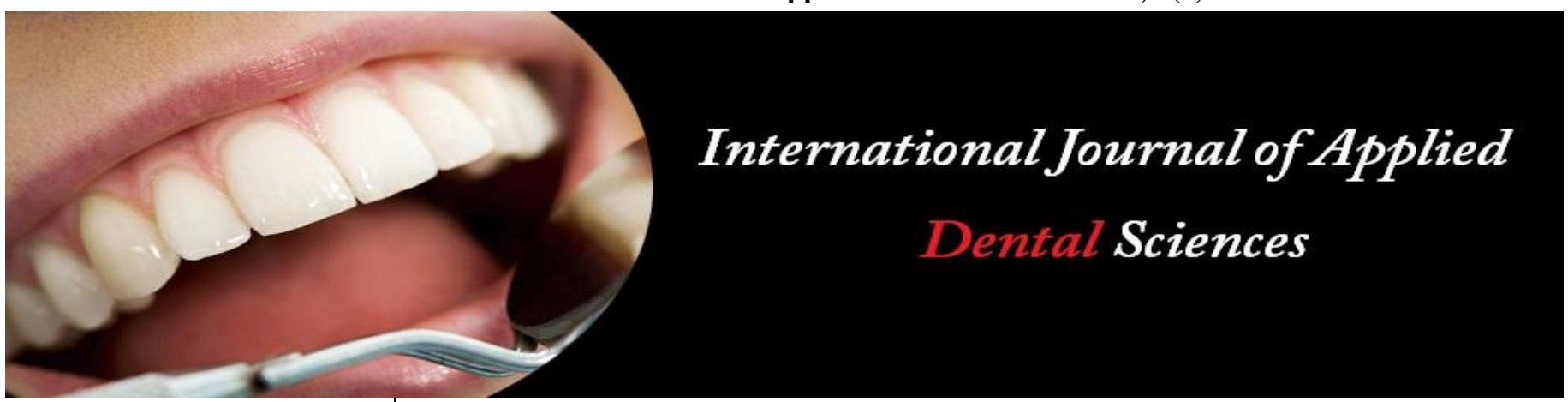

ISSN Print: 2394-7489

ISSN Online: 2394-7497

IJADS 2020; 6(4): 371-375

(C) 2020 IJADS

www.oraljournal.com

Received: 27-08-2020

Accepted: 12-10-2020

Luis-Martin Vargas-Zuñiga Doctor en Ciencias; Universidad Autónoma de Guerrero, Facultad de Odontología. Acapulco de Juárez de Guerrero, México

Hilda-Lourdes Muñuzuri-Arana Doctor en Ciencias; Universidad Autónoma de Guerrero, Facultad de Odontología. Acapulco de Juárez de Guerrero, México

Julio-Cesar Adams-Ocampo Maestria en Educacion; Universidad Autónoma de Guerrero, Facultad de Odontología. Acapulco de Juárez de Guerrero, México

Dora-Luz Merino-Rodriguez Cirujano Dentista; Universidad Autónoma de Guerrero, Facultad de Odontología. Acapulco de Juárez de Guerrero, México

Claudio Cabral-Romero

Doctor en Ciencias. Universidad

Autónoma de Nuevo León, Facultad de Odontología. Laboratorio de Biología Molecular. Monterrey, Nuevo Leon, Mexico

Rene Hernandez-Delgadillo

Doctor en Ciencias. Universidad

Autónoma de Nuevo León, Facultad de Odontología. Laboratorio de Biología Molecular. Monterrey, Nuevo Leon, Mexico

Gustavo-Israel Martinez-Gonzalez Doctor en Educacion. Universidad Autónoma de Nuevo León, Facultad de Odontología. Monterrey, Nuevo Leon, Mexico

Maria-Argelia-Akemi Nakagosi-Cepeda Doctor en Investigación. Universidad Autónoma de Nuevo León, Facultad de Odontología. Departamento de Histologia. Monterrey, Nuevo Leon, Mexico

Juan-Manuel Solis-Soto Doctor en Ciencias. Universidad Autónoma de Nuevo León. Facultad de Odontología. Departamento de Fisiologia. Monterrey, Nuevo Leon Mexico

\section{SARS-CoV-2, An odontological point of view}

Luis-Martin Vargas-Zuñiga, Hilda-Lourdes Muñuzuri-Arana, JulioCesar Adams-Ocampo, Dora-Luz Merino-Rodriguez, Claudio CabralRomero, Rene Hernandez-Delgadillo, Gustavo-Israel Martinez-Gonzalez, Maria-Argelia-Akemi Nakagosi-Cepeda and Juan-Manuel Solis-Soto

\section{DOI: https://doi.org/10.22271/oral.2020.v6.i4f.1090}

\section{Abstract}

Introduction: The epidemic of COVID-19 has become a major public health challenge for all countries around the world. For dental practices, strict and effective infection control protocols are imperative.

Objective: To evaluate the literature about SARS-CoV-2 from an odontological point of view.

Methodology: Using the keywords "COVID-19", "transmission routes", "serology", "diagnosis", "treatment", and "oral manifestations", the MEDLINE/PubMed and Science Direct databases were searched, from 2010 to 2020, with emphasis on the last 5 years. It was evaluated with the PRISMA and AMSTAR-2 guidelines. Results: The main transmission routes of the COVID-19 are direct contact, drops and air routes, and indirect one, fomite materials. Serological tests are complementary to molecular diagnosis in symptomatic patients, with their respective reservation, since false positives and negatives have been observed. The COVID-19 diagnosis could be made with saliva, since the salivary glands are the first affected by the SARS-CoV virus. So far, there is no specific treatment for COVID-19. Among the most important oral manifestations are anosmia, ageusia, and intraparotid lymphadenitis, which leads to a clinical picture similar to parotitis.

Conclusions: Information reviewed herein should be considered by the dentist in his daily practice, in order to reduce the risks of infection due to the frequent production of aerosol and the constant presence of saliva.

Keywords: COVID-19, SARS-CoV-2, coronavirus, oral, dental, saliva

\section{Introduction}

COVID-19 pandemic has resulted in millions of confirmed cases and thousands of deaths worldwide. It has also raised fears of an impending economic crisis and recession [1]. Infections caused by viruses generally trigger a powerful immune response that helps to contain the infection ${ }^{[2]}$.

Severe acute respiratory syndrome coronavirus 2 (SARS-CoV-2) is the causative agent of a life-threatening disease that is a major global public health problem ${ }^{[3]}$. It causes serious respiratory illnesses such as pneumonia and lung failure, it was first reported in Wuhan, the capital of Hubei, China, which likely originated from zoonotic coronaviruses, such as SARSCoV-2, that arose in $2002^{[4]}$. In December 2019, an unprecedented outbreak of pneumonia, SARS-CoV-2 was identified as the causative agent and it was later named COVID-19 by the World Health Organization ${ }^{[5]}$.

Considering the significance of morbidity/mortality as well as the economic impact on health and social costs produced by COVID-19, we carried out this bibliographic review in order to have updated and objective information from reliable sources of the routes of transmission, serology, diagnosis, treatment and oral manifestations of COVID-19. The information collected will contribute to improving the prevention practices of dental professionals, by identifying the early symptoms of SARS-CoV-2 infection in patients requiring dental intervention.

\section{Materials and methods}

Articles on the subject published in the MEDLINE/PubMed and Science Direct databases, from January 2010 to July 2020, were analyzed, with emphasis on the last 5 years. 
The quality of the articles was evaluated with the PRISMA guidelines (http://www.prisma-statement.org), that is, identification, review, choice and inclusion. The quality of the reviews was assessed with the Ameasurement Tool to Assess Systematic Reviews (AMSTAR-2) ${ }^{[6]}$.

The search was performed using logical Boolean operators and, OR and NOT.

The search was performed with the words "Covid-19" in conjunction with the words "transmission routes", "serology", "diagnosis", "treatment" and "oral manifestations".

\section{Results \& Discussion}

\subsection{Transmission Routes}

The importance of indirect contact transmission of Covid-19 (involving contamination from inanimate surfaces) is uncertain compared to other transmission routes. Animal and mathematical models, and intervention studies suggest that contact transmission is the most important route in some scenarios ${ }^{[7]}$.

Detection of the SARS-CoV-2 virus in faeces and wastewater has recently been reported, raising the hypothesis of fecal-oral transmission, this could have far-reaching consequences for public health and pandemic control strategies [8, 9]. The possibility of fecal-oral transmission of SARS-CoV-2 has implications, especially in areas with poor sanitation, so the importance of frequent and adequate hand hygiene should be emphasized ${ }^{[10]}$.

The available information on the spread of SARS-CoV-2 supports the hypothesis of airborne spread of infected droplets from person to person over a distance of more than two meters ${ }^{[11]}$. Direct transmission routes include cough, sneeze, droplet inhalation transmission, and contact transmission, such as the contact with oral, nasal, and eye mucous membranes. It can also be transmitted through the saliva, and the fetal-oral routes may also be a potential person-to-person transmission route ${ }^{[12]}$.

During the current Covid-19 pandemic, healthcare professionals are at the forefront of managing the highly infectious coronavirus, because the most common route of transmission is through aerosols and inhalation of drops, so it is essential that healthcare workers have the correct personal protective equipment, including gowns, masks, and goggles [13].

Covid-19 infection spreads mainly through direct contact with Flügge microdroplets or core drops that remain suspended in the form of an aerosol. Dentistry is one of the medical practices with the highest risk of infection due to the frequent production of aerosol and the constant presence of saliva ${ }^{[14]}$. The risk of cross-infection by SARS-CoV-2 could be high among dental specialists and patients due to the peculiarity of dental practice ${ }^{[15]}$.

The most important transmission route for Covid-19 infection is direct contact, drops and air routes, independent of the indirect one that occurs due to fomites that still remains unconfirmed.

\subsection{Serology}

There is much to be determined regarding the value of serological tests in the diagnosis and monitoring of Covid-19. However, more comprehensive evaluations of their performance are underway. The use of serology methods requires appropriate interpretations of the results and an understanding of the strengths and limitations of such tests [16].

Serum antibody testing is becoming one of the critical methods for diagnosing patients with Covid-19. IgM and IgG responses against nucleocapsid $(\mathrm{N})$ and spike $(\mathrm{S})$ protein have been investigated ${ }^{[17]}$. Low positive predictive values indicate that rapid Anhui and Clungene tests detecting the presence of anti-IgM and anti-IgG against SARS-CoV-2 virus infection are not suitable for screening the general population ${ }^{[18]}$. On the other hand, the presence of rheumatoid factor-IgM in medium to high levels could lead to false positive reactivity of SARS-CoV-2 IgM. Urea dissociation tests would be useful to reduce false IgM from SARS-CoV-2 positive results ${ }^{[19]}$.

To facilitate public health response, it is essential to have a conceptual framework, for assessing the sufficiency of patient-collected samples for the detection of SARS-CoV-2 RNA. Also, to provide personal protective equipment for healthcare workers collecting the nasopharyngeal swab specimens, and to have a special viral transport media for transporting the specimens ${ }^{[20]}$.

Molecular diagnosis of the disease is usually made through detection of nucleic acid-based virus from swabs, sputum, or bronchoalveolar lavage fluid. However, the positive rate of commonly used samples (swabs or sputum) is less than $75 \%$, mainly for insufficient viral RNA. Immunological assays for SARS-CoV-2 are needed to accurately diagnose Covid-19. Colloidal gold immunochromatographic assay was found to be a useful test to complement existing PCR-based assays for confirmation of Covid-19, as well as to assist in the surveillance of individuals with prior exposure to SARSCoV-2 [21, 22, 23].

Serological tests would be complementary to molecular diagnosis in patients with symptomatic Covid-19, but with reservation, since false positives and negatives have been observed, because the production of the antibodies is not $100 \%$ specific for the disease. On the other hand, they could be useful to monitor convalescent patients.

\subsection{Diagnosis}

Saliva could be an alternative sample for the diagnosis of Covid-19. The collection is non-invasive and does not generate aerosols. This method could facilitate the diagnosis of the disease, given the simplicity of sample collection and good diagnostic performance ${ }^{[24,25]}$. Saliva can play a critical role in person-to-person transmission, and non-invasive saliva diagnosis can provide a convenient and cost-effective pointof-care platform for early and rapid detection of Covid-19 infection ${ }^{[26]}$. Covid-19 can be detected by salivary diagnostic tests that have many advantages for healthcare professionals and patients ${ }^{[27]}$.

Salivary diagnosis is a dynamic field that is being incorporated as part of disease diagnosis, clinical monitoring of systemic health and to make meaningful clinical decisions for patient care. More research is required to analyze the potential diagnosis of Covid-19 in saliva to develop rapid tests for the detection of Covid-19 and it is also essential to improve and develop successful prevention strategies, especially for dentists and health professionals who are involved in performing aerosol-generating procedures ${ }^{[28]}$.

Saliva has been reported SARS-CoV-2 nucleic acid positive. Besides lungs, salivary glands and tongue are possibly another hosts of SARS-CoV-2 due to the expression of angiotensin-converting enzyme (ACE2 $(+)^{[29]}$. Cells positive for ACE2 (+) have been found to be widely distributed in the upper respiratory tract, and ACE2 (+) epithelial cells lining the salivary gland ducts were the first cells infected. These findings have implications for the early diagnosis and prevention of SARS-CoV-2 ${ }^{[30]}$. Since ACE2 (+) is expressed 
in the mucosa of the oral cavity, there is a potentially huge risk of infectious vulnerability. Samples analysis from the oral cavity presents an opportunity for the future prevention procedure in dental practice and daily life ${ }^{[31]}$.

Despite the fact that the diagnosis is made clinically and its confirmation is made through biological samples, it would be interesting to explore the use of saliva as a diagnostic sample of Covid-19, since it has been found that ACE2 (+) epithelial cells, lining the salivary gland ducts, are one of the first affected by the SARS-CoV-2.

\subsection{Treatment}

Treatments for Covid-19 include antiviral agents, chloroquine and hydroxychloroquine, corticosteroids, antibodies, convalescent plasma transfusions and potential vaccines ${ }^{[32]}$. SARS-CoV-2 induces excessive and prolonged cytokine/chemokine responses in some infected people. Timely control of the cytokine storm, in its initial stage, through means such as immunomodulators and cytokine antagonists, as well as the reduction of the infiltration of inflammatory lung cells, is the key to improve the treatment success rate and reduce the mortality rate of patients with Covid-19 ${ }^{[33]}$.

Currently, there is no specific effective antiviral treatment for Covid-19. Although most patients with Covid-19 have mild or moderate courses, up to 5\%-10\% may have a severe and lifethreatening course, there is an urgent need for effective medications ${ }^{[34]}$.

Recent publications have drawn attention to the possible benefit of chloroquine, a widely used antimalarial drug, in the treatment of patients infected. The scientific community should consider this information in light of previous experiments with chloroquine in the field of antiviral research ${ }^{[35]}$. Chloroquine and hydroxychloroquine are drugs that have shown in vitro activity in the replication of certain coronaviruses. In the absence of evidence of clinical benefit, the main risk is to unnecessarily expose patients to the adverse effects of these drugs, with a possibly increased risk in the specific setting of Covid-19. Furthermore, widespread use outside of any recommendation runs the risk of compromising good quality clinical trials ${ }^{[36]}$.

Monoclonal antibodies represent the main class of biotherapeutics for passive immunotherapy to fight viral infection. The therapeutic potential of monoclonal antibodies has been well recognized in the treatment of many diseases [37]. Convalescent plasma has also been used in the Covid-19 pandemic; limited data from China suggests clinical benefit, including radiological resolution, reduced viral loads, and better survival ${ }^{[38]}$.

The Covid-19 vaccine, considered an effective prophylactic strategy for control and prevention, is being developed in approximately 90 institutions worldwide. The experiences and lessons found in previous SARS and MERS vaccine research can be used as a reference in the development of the Covid-19 vaccine $^{[39]}$.

So far, there is no specific treatment for Covid-19, despite the fact that antiviral agents, antimalarial drugs such as chloroquine and hydroxychloroquine, as well as immunomodulators and antagonists of cytokines, antibodies, transfusions of convalescent plasmas and experimental vaccines produced by recombinant RNA.

\subsection{Oral Manifestations}

Covid-19 can affect the oral health of older people, due to the high risk of viral infection, which together with neglected dental conditions can worsen their oral health, causing infections that can lead to local and systemic complications, compromising general health ${ }^{[40]}$. Olfactory and gustatory disorders are prevalent symptoms in European patients with Covid-19, who may not have nasal symptoms. Sudden anosmia or ageusia should be recognized by the international scientific community as important symptoms of Covid-19 infection ${ }^{[41]}$.

Ear, nose, and throat symptoms may precede the development of severe Covid-19. During the Covid-19 pandemic, patients with cough, sore throat, dyspnea, hyposmia/anosmia, and a history of travel to the region with confirmed infected patients, should be considered as possible cases of Covid-19 $[42,43]$.

Olfactory dysfunction is highly prevalent during Covid-19, occurs early, and is very severe, often in conjunction with loss of flavor. It is negatively associated with advanced age and positively with female sex. Olfactory dysfunction patients may also experience more severe shortness of breath ${ }^{[44]}$.

Clinical characteristics of three patients in France who had parotitis have been reported, as a clinical manifestation of confirmed coronavirus disease. Magnetic resonance imaging results support the appearance of intraparotid lymphadenitis, leading to a clinical picture similar to parotitis ${ }^{[45]}$. SARSCoV-2 is speculated to enter via salivary glands ${ }^{[46]}$. It causes oral ulceration and blisters, which often are undetected due to a lack of intraoral examination during hospital admission ${ }^{[47]}$.

Anosmia associated or not with dysgeusia seems to be a frequent symptom in cases of SARS-CoV-2 infection responsible for Covid-19. It can be the initial symptom of the disease or remain isolated in pauci-symptomatic patients. Pending scientific confirmation and in the context of the current pandemic, it seems essential to consider any patient with a new anosmia as infected with SARS-CoV-2 until proven otherwise ${ }^{[48]}$.

The clinical manifestations are varied, from an asymptomatic state to the acute respiratory distress syndrome and multiorgan dysfunction, regarding the oral cavity, there are anosmia or ageusia, as well as the appearance of intraparotid lymphadenitis, which leads to a clinical picture similar to parotitis, among the most important.

\section{Conclusions}

That Covid-19 is a highly contagious disease, its main route of transmission is through oral secretions, serological tests are complementary to molecular tests of the virus; its diagnosis could be made with saliva, although it requires more research. There is no specific treatment and the oral manifestations that occur are anosmia or ageusia, as well as a clinical picture similar to parotitis, but not only in Covid-19, but in most respiratory conditions of viral origin.

\section{References}

1. Nicola M, Alsafi Z, Sohrabi C, Kerwan A, Al-Jabir A, Iosifidis $\mathrm{C}$ et al. The socio-economic implications of the coronavirus pandemic (COVID-19): A review. Int J Surg. 2020;78:185-193.

2. Hussein HM, Rahal EA. The role of viral infections in the development of autoimmune diseases. Crit Rev Microbiol. 2019;45(4):394-412.

3. Rothan HA, Byrareddy SN. The epidemiology and pathogenesis of coronavirus disease (COVID-19) outbreak. J Autoimmun. 2020;109:102433.

4. Ahn DG, Shin HJ, Kim MH, Lee S, Kim HS, Myoung J et al. Current Status of Epidemiology, Diagnosis, 
Therapeutics, and Vaccines for Novel Coronavirus Disease 2019 (COVID-19). J Microbiol Biotechnol. 2020;30(3):313-324.

5. Sohrabi C, Alsafi Z, O'Neill N, Khan M, Kerwan A, AlJabir A et al. World Health Organization declares global emergency: A review of the 2019 novel coronavirus (COVID-19). Int J Surg. 2020;76:71-76.

6. Shea BJ, Reeves BC, Wells G, Thuku M, Hamel C, Moran $\mathrm{J}$ et al. AMSTAR 2: a critical appraisal tool for systematic reviews that include randomised or nonrandomised studies of healthcare interventions, or both. BMJ. 2017;358:j4008.

7. Otter JA, Donskey C, Yezli S, Douthwaite S, Goldenberg SD, Weber DJ. Transmission of SARS and MERS coronaviruses and influenza virus in healthcare settings: the possible role of dry surface contamination. J Hosp Infect. 2016;92(3):235-250.

8. Heller L, Mota CR, Greco DB. COVID-19 faecal-oral transmission: Are we asking the right questions? Sci Total Environ. 2020;729:138919.

9. Yang C, Ma QY, Zheng YH, Yang YX. Transmission Routes of 2019-novel Coronavirus (2019-nCoV). Zhonghua Yu Fang Yi Xue Za Zhi. 2020;54 (4):374-377.

10. Yeo C, Kaushal S, Yeo D. Enteric involvement of coronaviruses: is faecal-oral transmission of SARS-CoV2 possible?. Lancet Gastroenterol Hepatol. 2020;5(4):335-337.

11. Setti L, Passarini F, De Gennaro G, Barbieri P, Perrone MG, Borelli M et al. Airborne Transmission Route of COVID-19: Why 2 Meters/6 Feet of Inter-Personal Distance Could Not Be Enough. Int J Environ Res Public Health. 2020;17(8):2932.

12. Peng $\mathrm{X}, \mathrm{Xu} \mathrm{X}$, Li Y, Cheng L, Zhou $\mathrm{X}$, Ren $\mathrm{B}$. Transmission routes of 2019-nCoV and controls in dental practice. Int J Oral Sci. 2020;12(1):9.

13. Umer F, Haji Z, Zafar $K$. Role of respirators in controlling the spread of novel coronavirus (COVID-19) amongst dental healthcare providers: a review. Int Endod J. 2020:10.1111/iej.13313.

14. Lo Giudice R. The Severe Acute Respiratory Syndrome Coronavirus-2 (SARS CoV-2) in Dentistry. Management of Biological Risk in Dental Practice. Int J Environ Res Public Health. 2020;17(9):3067.

15. Pan Y, Liu H, Chu C, Li X, Liu S, Lu S. Transmission routes of SARS-CoV-2 and protective measures in dental clinics during the COVID-19 pandemic. Am J Dent. 2020;33(3):129-134.

16. Zainol Rashid Z, Othman SN, Abdul Samat MN, Ali UK, Wong KK. Diagnostic performance of COVID-19 serology assays. Malays J Pathol. 2020;42(1):13-21.

17. Sun B, Feng Y, Mo X, Zheng $\mathrm{P}$, Wang Q, Li $\mathrm{P}$ et al. Kinetics of SARS-CoV-2 specific IgM and IgG responses in COVID-19 patients. Emerg Microbes Infect. 2020;9(1):940-948.

18. Vásárhelyi B, Kristóf K, Ostorházi E, Szabó D, Prohászka Z, Merkely B. The diagnostic value of rapid anti $\operatorname{IgM}$ and $\mathrm{IgG}$ detecting tests in the identification of patients with SARS CoV-2 virus infection. Orv Hetil. 2020;161(20):807-812.

19. Wang Q, Du Q, Guo B, Mu D, Lu X, Ma Q et al. A Method To Prevent SARS-CoV-2 IgM False Positives in Gold Immunochromatography and Enzyme-Linked Immunosorbent Assays. J Clin Microbiol. 2020:58(6):e00375-20.

20. Sullivan PS, Sailey C, Guest JL, Guarner J, Kelley C,
Siegler AJ et al. Detection of SARS-CoV-2 RNA and Antibodies in Diverse Samples: Protocol to Validate the Sufficiency of Provider-Observed, Home-Collected Blood, Saliva, and Oropharyngeal Samples. JMIR Public Health Surveill. 2020 24;6(2):e19054.

21. Shen L, Wang C, Zhao J, Tang X, Shen Y, Lu M et al. Delayed specific IgM antibody responses observed among COVID-19 patients with severe progression. Emerg Microbes Infect. 2020;9(1):1096-1101.

22. Lee CY, Lin RTP, Renia L, Ng LFP. Serological Approaches for COVID-19: Epidemiologic Perspective on Surveillance and Control. Front Immunol. 2020;11:879.

23. Yongchen Z, Shen H, Wang X, Shi X, Li Y, Yan J et al. Different longitudinal patterns of nucleic acid and serology testing results based on disease severity of COVID-19 patients. Emerg Microbes Infect. 2020;9(1):833-836.

24. Khurshid Z, Asiri FYI, Al Wadaani H. Human Saliva: Non-Invasive Fluid for Detecting Novel Coronavirus (2019-nCoV). Int J Environ Res Public Health. 2020;17(7):2225.

25. Pasomsub E, Watcharananan SP, Boonyawat K, Janchompoo P, Wongtabtim G, Suksuwan W et al. Saliva sample as a non-invasive specimen for the diagnosis of coronavirus disease 2019: a cross-sectional study. Clin Microbiol Infect. 2020:S1198-743X(20)30278-0.

26. Sabino-Silva R, Jardim ACG, Siqueira WL. Coronavirus COVID-19 impacts to dentistry and potential salivary diagnosis. Clin Oral Investig. 2020;24 (4):1619-1621.

27. Baghizadeh Fini M. Oral saliva and COVID-19. Oral Oncol. 2020;108:104821.

28. Sri Santosh T, Parmar R, Anand H, Srikanth K, Saritha M. A Review of Salivary Diagnostics and Its Potential Implication in Detection of Covid-19. Cureus. 2020;12(4):e7708.

29. Xu R, Cui B, Duan X, Zhang P, Zhou X, Yuan Q. Saliva: potential diagnostic value and transmission of 2019nCoV. Int J Oral Sci. 2020;12(1):11.

30. Liu L, Wei Q, Alvarez X, Wang H, Du Y, Zhu H et al. Epithelial cells lining salivary gland ducts are early target cells of severe acute respiratory syndrome coronavirus infection in the upper respiratory tracts of rhesus macaques. J Virol. 2011;85(8):4025-30.

31. Baghizadeh Fini M. What dentists need to know about COVID-19. Oral Oncol. 2020;105:104741.

32. Zhai P, Ding Y, Wu X, Long J, Zhong Y, Li Y. The epidemiology, diagnosis and treatment of COVID-19. Int J Antimicrob Agents. 2020;55(5):105955.

33. Ye Q, Wang B, Mao J. The pathogenesis and treatment of the 'Cytokine Storm' in COVID-19. J Infect. 2020;80(6):607-613.

34. Şimşek Yavuz S, Ünal S. Antiviral treatment of COVID19. Turk J Med Sci. 2020;50(SI-1):611-619.

35. Touret F, de Lamballerie X. Of chloroquine and COVID19. Antiviral Res. 2020;177:104762.

36. Roustit M, Guilhaumou R, Molimard M, Drici MD, Laporte S, Montastruc JL. French Society of Pharmacology and Therapeutics (SFPT). Chloroquine and hydroxychloroquine in the management of COVID19: Much kerfuffle but little evidence. Therapie. 2020;S0040-5957(20):30100-1.

37. Shanmugaraj B, Siriwattananon K, Wangkanont K, Phoolcharoen W. Perspectives on monoclonal antibody therapy as potential therapeutic intervention for 
Coronavirus disease-19 (COVID-19). Asian Pac J Allergy Immunol. 2020;38(1):10-18.

38. Bloch EM, Shoham S, Casadevall A, Sachais BS, Shaz B, Winters JL et al. Deployment of convalescent plasma for the prevention and treatment of COVID-19. J Clin Invest. 2020;130(6):2757-2765.

39. Yang L, Tian D, Liu W. Strategies for vaccine development of COVID-19. Sheng Wu Gong Cheng Xue Bao. 2020;36(4):593-604.

40. León S, Giacaman RA. COVID-19 and Inequities in Oral Health Care for Older People: An Opportunity for Emerging Paradigms. JDR Clin Trans Res. 2020,2380084420934742.

41. Lechien JR, Chiesa-Estomba CM, De Siati DR, Horoi M, Le Bon SD, Rodriguez A et al. Olfactory and gustatory dysfunctions as a clinical presentation of mild-tomoderate forms of the coronavirus disease (COVID-19): a multicenter European study. Eur Arch Otorhinolaryngol. 2020;277(8):2251-2261.

42. Krajewska J, Krajewski W, Zub K, Zatoński T. COVID19 in otolaryngologist practice: a review of current knowledge. Eur Arch Otorhinolaryngol. 2020;277(7):1885-1897.

43. Herrera D, Serrano J, Roldán S, Sanz M. Is the oral cavity relevant in SARS-CoV-2 pandemic? Clin Oral Investig. 2020;24(8):2925-2930.

44. Speth MM, Singer-Cornelius T, Obere M, Gengler I, Brockmeier SJ, Sedaghat AR. Olfactory Dysfunction and Sinonasal Symptomatology in COVID-19: Prevalence, Severity, Timing, and Associated Characteristics. Otolaryngol Head Neck Surg. 2020;194599820929185.

45. Lechien JR, Chetrit A, Chekkoury-Idrissi Y, Distinguin L, Circiu M, Saussez S et al. Parotitis-Like Symptoms Associated with Covid-19. Emerg Infect Dis. 2020;26(9):2270-2271.

46. Song J, Li Y, Huang X, Chen Z, Li Y, Liu C et al. Systematic analysis of ACE2 and TMPRSS2 expression in salivary glands reveals underlying transmission mechanism caused by SARS-CoV-2. J Med Virol. 2020:10.1002/jmv.26045.

47. Santos JAD, Normando AGC, Silva RLCD, Paula RM, Cembranel AC, Santos-Silva AR et al. Oral mucosal lesions in a COVID-19 patient: new signs or secondary manifestations? Int J Infect Dis. 2020;97:326-8.

48. Reinhard A, Ikonomidis C, Broome M, Gorostidi F. Anosmia and COVID-19. Rev Med Suisse. 2020;16(N ${ }^{\circ}$ 691-2):849-851 